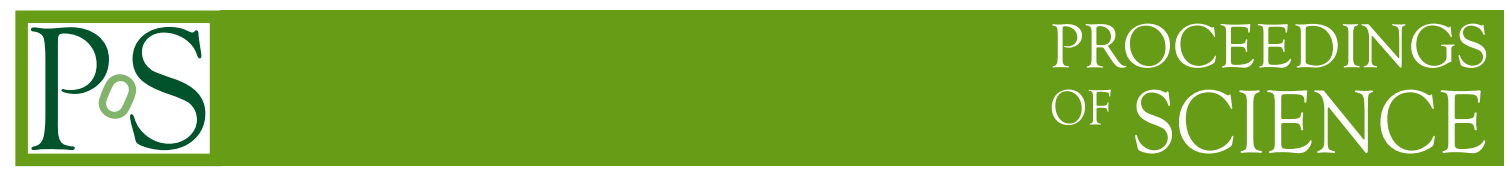

\title{
Probing properties of nuclear matter with HADES
}

Jerzy Pietraszko for the HADES collaboration* ${ }^{*}$

Institut für Kernphysik, Goethe-Universität, 60438 Frankfurt, Germany

E-mail: G.pietraszkodgsi.de

G. Agakishiev ${ }^{6}$, A. Balanda ${ }^{3}$, D. Belver ${ }^{16}$, A. Belyaev ${ }^{6}$, A. Blanco ${ }^{2}$, M. Böhmer ${ }^{9}$, J. L. Boyard ${ }^{14}$, P. Cabanelas ${ }^{16}$, E. Castro ${ }^{16}$, S. Chernenko ${ }^{6}$, J. Díaz ${ }^{17}$, A. Dybczak ${ }^{3}$, E. Epple ${ }^{8}$, L. Fabbietti ${ }^{8}$, O. Fateev ${ }^{6}$, P. Finocchiaro ${ }^{1}$, P. Fonte ${ }^{2, b}$, J. Friese ${ }^{9}$, I. Fröhlich ${ }^{7}$, T. Galatyuk ${ }^{7, c}$, J. A. Garzón ${ }^{16}$, A. Gil ${ }^{17}$, M. Golubeva ${ }^{12}$, D. González-Díaz ${ }^{d}$, F. Guber ${ }^{12}$, M. Gumberidze ${ }^{14}$, T. Hennino ${ }^{14}$, R. Holzmann ${ }^{4}$, P. Huck ${ }^{9}$, A. lerusalimov ${ }^{6}$, I. Iori ${ }^{11, f}$, A. Ivashkin ${ }^{12}$, M. Jurkovic ${ }^{9}$, B. Kämpfer ${ }^{5, e}$, T. Karavicheva ${ }^{12}$, I. Koenig ${ }^{4}$, W. Koenig ${ }^{4}$, B. W. Kolb ${ }^{4}$, G. Korcyl ${ }^{3}$, G. Kornakov ${ }^{16}$, R. Kotte ${ }^{5}$, A. Kozuch ${ }^{3, g}$, A. Krása ${ }^{15}$, F. Krizek ${ }^{15}$, R. Krücken ${ }^{9}$, H. Kuc ${ }^{3,14}$, W. Kühn ${ }^{10}$, A. Kugler ${ }^{15}$, A. Kurepin ${ }^{12}$, A. Kurilkin ${ }^{6}$, P. Kurilkin ${ }^{6}$, P. Kählitz ${ }^{5}$, V. Ladygin ${ }^{6}$, J. Lamas-Valverde ${ }^{16}$, S. Lang ${ }^{4}$, K. Lapidus ${ }^{8}$, T. Liu' ${ }^{14}$, L. Lopes ${ }^{2}$, M. Lorenz ${ }^{7}$, L. Maier ${ }^{9}$, A. Mangiarotti ${ }^{2}$, J. Markert ${ }^{7}$, V. Metag $^{10}$, B. Michalska ${ }^{3}$, J. Michel ${ }^{7}$, C. Müntz ${ }^{7}$, L. Naumann ${ }^{5}$, Y. C. Pachmayer ${ }^{7}$, M. Palka ${ }^{7}$, Y. Parpottas ${ }^{13}$, V. Pechenov ${ }^{4}$, O. Pechenova ${ }^{7}$, J. Pietraszko ${ }^{7}$, W. Przygoda ${ }^{3}$, B. Ramstein ${ }^{14}$, A. Reshetin ${ }^{12}$, J. Roskoss ${ }^{10}$, A. Rustamov ${ }^{4}$, A. Sadovsky ${ }^{12}$, P. Salabura ${ }^{3}$, A. Schmah ${ }^{a}$, J. Siebenson ${ }^{8}$, Yu.G. Sobolev ${ }^{15}$, S. Spataro ${ }^{h}$, H. Ströbele ${ }^{7}$, J. Stroth ${ }^{7,4}$, C. Sturm ${ }^{4}$,

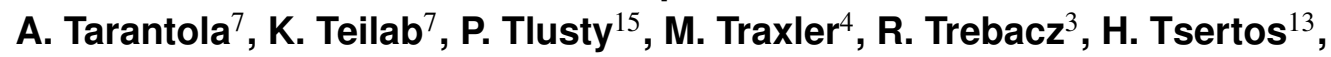
T. Vasiliev ${ }^{6}$, V. Wagner ${ }^{15}$, M. Weber ${ }^{9}$, J. Wüstenfeld ${ }^{5}$, S. Yurevich ${ }^{4}$, Y. Zanevsky $^{6}$ (HADES collaboration) 


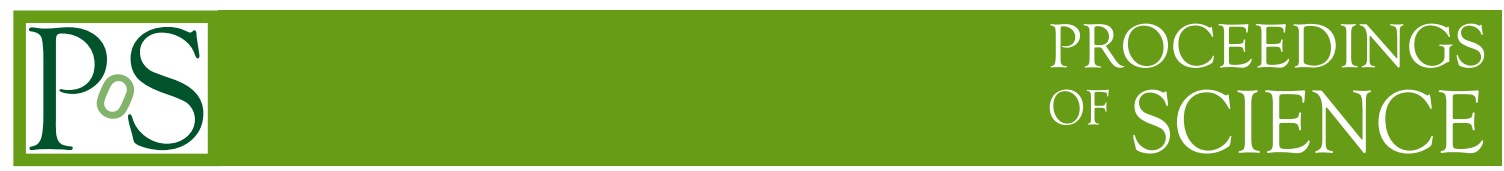

${ }^{1}$ Istituto Nazionale di Fisica Nucleare - Laboratori Nazionali del Sud, 95125 Catania, Italy

${ }^{2}$ LIP-Laboratório de Instrumentação e Física Experimental de Partículas , 3004-516 Coimbra, Portugal

${ }^{3}$ Smoluchowski Institute of Physics, Jagiellonian University of Cracow, 30-059 Kraków, Poland

${ }^{4}$ GSI Helmholtzzentrum für Schwerionenforschung GmbH, 64291 Darmstadt, Germany

${ }^{5}$ Institut für Strahlenphysik, Forschungszentrum Dresden-Rossendorf, 01314 Dresden, Germany

${ }^{6}$ Joint Institute of Nuclear Research, 141980 Dubna, Russia

${ }^{7}$ Institut für Kernphysik, Goethe-Universität, 60438 Frankfurt, Germany

${ }^{8}$ Excellence Cluster 'Origin and Structure of the Universe', 85748 Garching, Germany

${ }^{9}$ Physik Department E12, Technische Universität München, 85748 Garching, Germany

${ }^{10}$ II.Physikalisches Institut, Justus Liebig Universität Giessen, 35392 Giessen, Germany

${ }^{11}$ Istituto Nazionale di Fisica Nucleare, Sezione di Milano, 20133 Milano, Italy

${ }^{12}$ Institute for Nuclear Research, Russian Academy of Science, 117312 Moscow, Russia

${ }^{13}$ Department of Physics, University of Cyprus, 1678 Nicosia, Cyprus

${ }^{14}$ Institut de Physique Nucléaire (UMR 8608), CNRS/IN2P3 - Université Paris Sud, F-91406 Orsay Cedex, France

${ }^{15}$ Nuclear Physics Institute, Academy of Sciences of Czech Republic, 25068 Rez, Czech Republic

${ }^{16}$ Departamento de Física de Partículas, Univ. de Santiago de Compostela, 15706 Santiago de Compostela, Spain

${ }^{17}$ Instituto de Física Corpuscular, Universidad de Valencia-CSIC, 46971 Valencia, Spain

a also at Lawrence Berkeley National Laboratory, Berkeley, USA

${ }^{b}$ also at ISEC Coimbra, Coimbra, Portugal

${ }^{c}$ also at ExtreMe Matter Institute EMMI, 64291 Darmstadt, Germany

${ }^{d}$ also at Technische Univesität Darmstadt, Darmstadt, Germany

${ }^{e}$ also at Technische Universität Dresden, 01062 Dresden, Germany

${ }^{f}$ also at Dipartimento di Fisica, Università di Milano, 20133 Milano, Italy

${ }^{g}$ also at Panstwowa Wyzsza Szkola Zawodowa, 33-300 Nowy Sacz, Poland

${ }^{h}$ also at Dipartimento di Fisica Generale, Università di Torino, 10125 Torino, Italy

This paper presents a review of selected physics results obtained at GSI Helmholtzzentrum für Schwerionenforschung GmbH by HADES (High-Acceptance Di-Electron Spectrometer). The $e^{+} e^{-}$pair emission measured for proton and heavy-ion induced collisions is reported together with results on strangeness production. The future HADES activities at the planned FAIR facility are also discussed.

XLIX International Winter Meeting on Nuclear Physics, BORMIO2011

January 24-28, 2011

Bormio, Italy

* Speaker.

$\dagger$ 


\section{Introduction}

The study of in-medium properties of hadrons and, in general, the properties of nuclear matter at high densities and temperatures is one of the main objectives in relativistic heavy-ion physics. It is expected that the vacuum-properties of a single hadron are modified once it is placed in nuclear matter. To explore these phenomena an appropriate experimental approach, sensitive to the in-medium effects, and a quantitative theoretical treatment describing the change of hadron properties in the nuclear medium are essential. The low mass vector mesons $\rho, \omega$ have been suggested as one of the suitable probes for studying in-medium hadron modifications, because of their short life time. Additionally, the decays of vector mesons into di-electrons offers a unique experimental way to learn about the in-medium modifications, as the di-electrons carry out undisturbed information from the dense and hot nuclear matter. On the other hand, electromagnetic signals are very difficult to reconstruct, mainly due to the huge hadron background and low branching ratio of the electromagnetic channels. The dileptons measured in the detection system encode information about all emission processes integrated over the whole collision history. With precise knowledge of particle yields at chemical freeze-out, obtained from hadronic observables, it is however possible to subtract the late contributions from the pair spectrum. Using elementary collisions one can learn about contribution from first-chance collisions. Finally, the in-medium component can be reconstructed. Another, very attractive experimental probe to study the high density phase of nuclear matter are strange particles. In particular, from the subthreshold $\mathrm{K}^{+}$production tight constraints can be put on the nuclear equation of state at matter densities of 2-3 $\rho_{0}$ [四]. Furthermore, it has been demonstrated that the kaon phase space distributions and flow patterns are sensitive to the in-medium kaon potential [四]. The SIS energy regime (incident energies around $1 \mathrm{~A} \mathrm{GeV}$ ) is very interesting because pions are mainly produced in direct NN collisions, whereas kaons are dominantly produced via collective effects which are challenging for theoretical description. In elementary NN collisions strange particle production is well understood in contrast to heavy-ion reactions where multi-step processes play an extraordinary role.

The High Acceptance Di-Electron Spectrometer (HADES) [B] located at the heavy-ion synchrotron SIS18 at GSI Helmholzzentrum für Schwerionenforschung in Darmstadt, Germany, offers a unique capability of performing high quality di-electron and hadron identification with very good performance of vertex reconstruction, which is essential for measuring off-vertex decays. Thus, the HADES group has been actively participating in the study of nuclear matter properties by means of di-electron measurements, as well as the strange particle production.

This report reviews the recent HADES experimental findings in the dilepton and hadron section and is organized as follows: In section 2 the HADES Spectrometer is described. Section 3 focuses on dilepton pair production in heavy-ion and proton induced reactions. Section 4 reports on strangeness production measured with HADES. The future HADES at FAIR (SIS100) experiments are described in section 5. Finally, the paper is summarized in section 6.

\section{HADES Spectrometer}

HADES is a di-lepton spectrometer specially designed to operate at the 1-2 AGeV heavy ion synchrotron facility of GSI, Darmstadt and is described in details in reference [B]. In the design 
of the spectrometer, particular emphasis has been given to a good mass resolution $\left(\delta M_{i n v} / M_{i n v} \simeq\right.$ $1 \%(\sigma)$ ), an electron-positron, proton and pion identification capability over a broad momentum range $(0.1<\mathrm{p}<1.5 \mathrm{GeV} / \mathrm{c})$ and a large geometrical acceptance $\left(\Omega_{\text {pair }} \simeq 35 \%\right.$ of full solid angle at $\left.\mathrm{M}_{\text {pair }} 0.5 \mathrm{GeV}\right)$.

A cross section of the detector configuration is shown in Fig. $\mathrm{W}$. Beam ions are incident on a segmented target located in the center of a gaseous Ring Imaging Čerenkov (RICH) detector used for $e^{+} / e^{-}$identification. The spectrometer consists of a toroidal magnet with 6 coils centered on the beam axis and six identical detection sections mounted between the coils which cover polar angles from $18^{\circ}$ to $85^{\circ}$. These detection sections are used to detect and track reaction products from the nuclear collision zone with a set of Multiwire Drift Chambers (MDCs) and a Multiplicity Electron Trigger Array (META) which consists of a segmented Time Of Flight wall and a PreSHOWER detector covering forward polar angles.

Particle tracking and momentum determination are performed by four planes of MDCs, two of them located in front (inner MDCs modules) and two behind the magnetic field (outer MDCs modules).

The charged particle identification procedure combines the information from the tracking detectors (momentum, dE/dx) and META measurements (Time-of-Flight, dE/dx). The RICH detector, composed of a gas radiator, a spherical mirror and a position sensitive photon detector, serves as the central lepton identification device. At SIS beam energies the RICH detector is hadron blind due to the low Čerenkov threshold ( $\gamma_{t h}=18.3$ ) of the $\mathrm{C}_{4} \mathrm{~F}_{10}$ radiator gas.

Due to the small vector meson production cross sections and low branching ratios for $e^{+} e^{-}$decays $\left(10^{-4}-10^{-5}\right)$ a two-level selective trigger is used in HADES. The first-level trigger LVL1 is derived from a fast charged particle multiplicity measurement in the TOF detectors in coincidence with a fast timing signal from a in-beam diamond detector located $70 \mathrm{~cm}$ upstream of the target. A second-level trigger, based on online matching of hits from leptons in the RICH detector and in the META detector is used to enhance the di-electron statistics. The attained enhancement in di-lepton contents per event is of up to one order of magnitude.

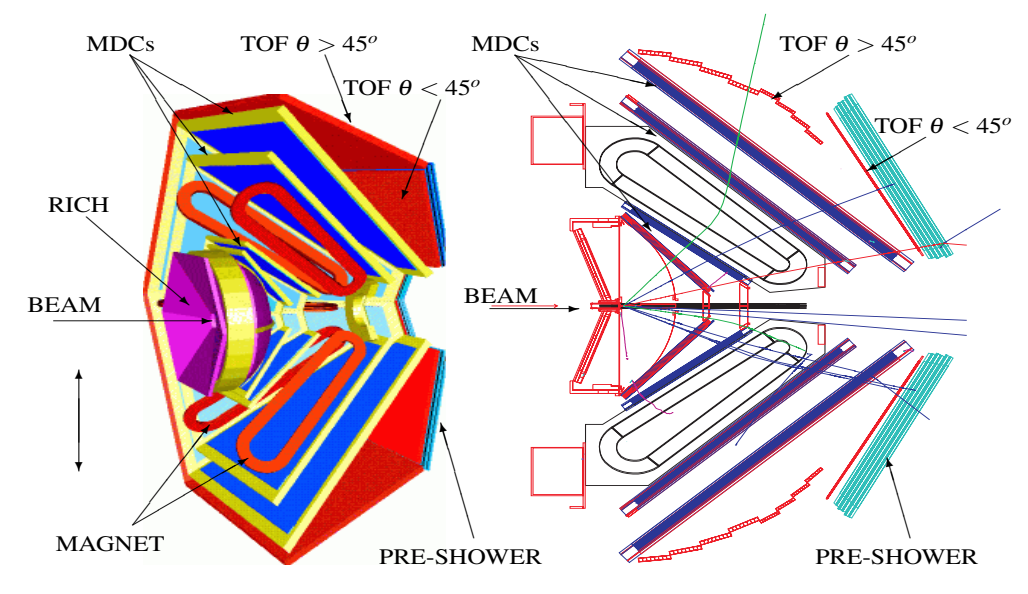

Figure 1: Layout of the HADES Spectrometer. In the left-hand picture two of the six sectors have been removed for clarity. On the right-hand side the azimuthal cross section of the HADES setup is shown. 


\section{Di-electron probes}

The observation of an enhanced yield of low-mass lepton pairs $\left(\mathrm{M}_{\text {pair }}<1 \mathrm{GeV} / \mathrm{c}^{2}\right)$ extends back over more than two decades. The enhanced yield, defined as the excess above the contribution expected from well known hadronic sources, was reported by several groups for reactions in a large range of bombarding energies. Starting in the Bevalac energy regime (1-2 A GeV) with the

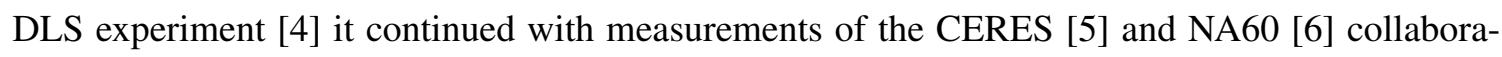
tions at low and top SPS energies. While the excess observed at SPS energies could fairly well be described by different transport models involving a significant in-medium modification of the rho meson spectral function in the hadronic medium [ $[$ ], the results obtained at lower beam energies of 1-2 A GeV [四] could not be satisfactorily reproduced by theoretical models which led to the so-called "DLS-puzzle" [8]].

\subsection{Low- and intermediate-mass region}

The HADES collaboration has therefore started a systematic investigation of dilepton production in the SIS18 energy regime of 1-2 A GeV. In the beginning the focus was on light collision systems to revisit the "DLS-puzzle". In order to verify the DLS findings an experiment was conducted in which a $1 \mathrm{~A} \mathrm{GeV}{ }^{12} \mathrm{C}$ beam of $10^{6}$ particles/s was incident onto a carbon target with a thickness corresponding to $4.6 \%$ of the nuclear interaction length [Q]. The measured inclusive di-electron production in the invariant pair mass region $0.15 \mathrm{GeV} / \mathrm{c}^{2}<\mathrm{M}_{e e}<0.50 \mathrm{GeV} / \mathrm{c}^{2}$ was considerably stronger than expected from the contribution of $\eta$ meson decays. A comprehensive comparison of the HADES and DLS results was achieved by a procedure taking the very different acceptances and trigger conditions of both experiments [Q] into account. The resulting differential cross sections for invariant pair masses and transverse momenta are depicted in figure $\square$ and exhibit a good agreement of both measurements indicating that indeed the theoretical interpretation of the data based on model descriptions needs a better understanding of the processes important at this beam energy i.e. coupling of the $\rho$ meson to $\mathrm{N}^{\star}$.

To gain further insight the HADES group performed measurements of inclusive di-electron production in $\mathrm{C}+\mathrm{C}$ at $2 \mathrm{~A} \mathrm{GeV} \mathrm{[ \amalg ]]} \mathrm{and} \mathrm{in} \mathrm{Ar}+\mathrm{KCl}$ at $1.76 \mathrm{~A} \mathrm{GeV}$ [ए]].

For both reaction systems a significant excess of di-electron yield above the known contributions has been extracted. In order to compensate for different trigger settings, which influence the centrality selection in various collision systems, all measured yields were extrapolated to the full solid angle and were normalized to their respective average multiplicity of $\mathrm{A}_{\text {part }}$ of participating nucleons. Figure 3 shows for all available reaction systems a compilation of the respective pair yields integrated over the mass region $\mathrm{M}_{e e}=0.15-0.5 \mathrm{GeV} / \mathrm{c}^{2}$ and exceeding expected $\eta$ - and vector meson contributions. The figure contains also the TAPS results on inclusive $\pi^{0}$ and $\eta$ multiplicities measured in photon calorimetry [12], [13]]. The resulting excitation function of meson and di-electron production for light and medium mass systems leads to the conclusion that the low mass di-electron excess yields show a similar trend as the measured pion multiplicities. This hints to an important role of low-energy processes like pion production and propagation involving the $\Delta$ resonance as well as NN bremsstrahlung [14]]. A strong virtual bremsstrahlung contribution to the pair yield mainly from $n+p$ interactions was suggested by effective model calculations done 

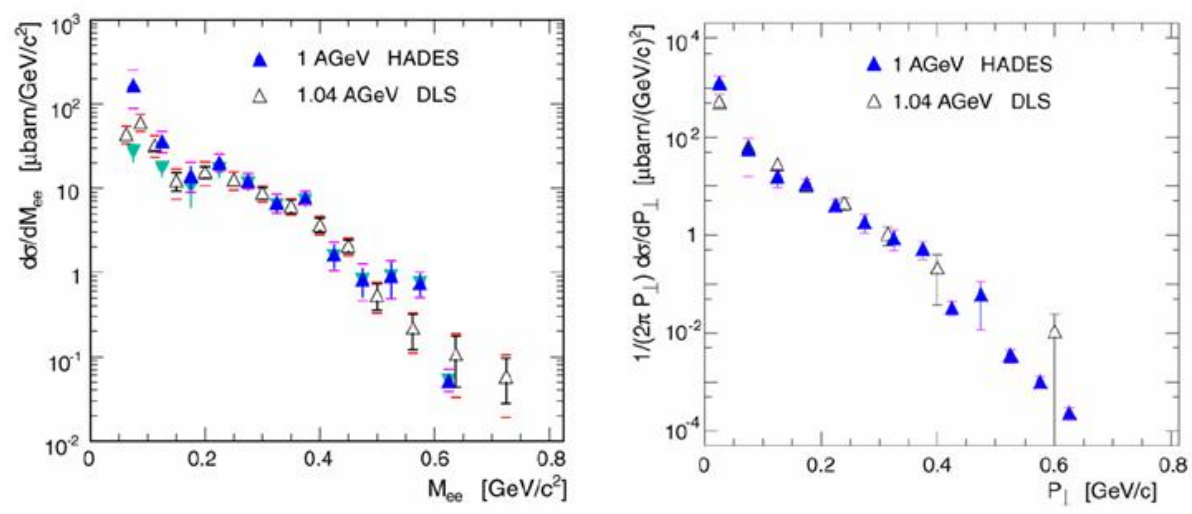

Figure 2: Dilepton cross sections measured in $\mathrm{C}+\mathrm{C}$ at $1 \mathrm{~A} \mathrm{GeV}$ by HADES and at $1.04 \mathrm{~A} \mathrm{GeV}$ by DLS, as a function of invariant mass (left panel) and transverse momentum (right frame). Both distributions are shown within the DLS acceptance to allow for direct comparison. Analysis details are given in [Q]].

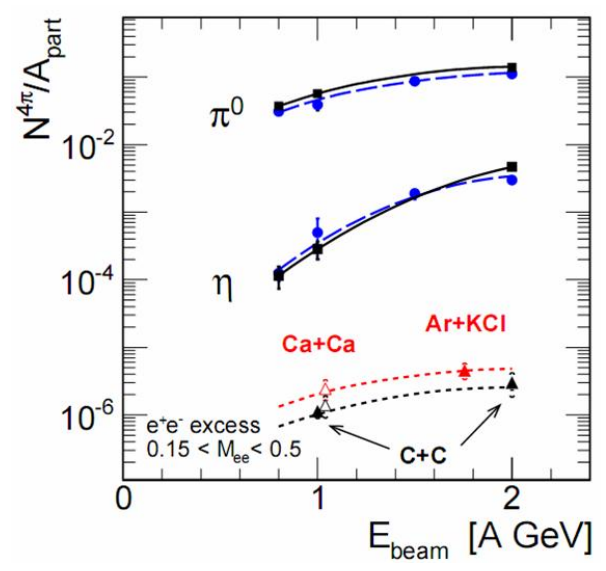

Figure 3: Multiplicity of excess yield per participant as a function of beam energy for HADES Ar $+\mathrm{KCl}$ and C + C data [四, 四] as well as for DLS data [四]. The $\pi^{0}$ and $\eta$ multiplicities from TAPS measurements [2, [3] are shown. For easier visual comparison the multiplicity of excess yields are overlaid with the down-scaled $\pi^{0}$ multiplicity. For details see text and [U]].

by various authors $[\mathbb{[ 1 5},[6]$. The HADES high quality results on dilepton production in $\mathrm{p}+\mathrm{p}$ and $\mathrm{d}+\mathrm{p}$ interactions at $\mathrm{E}_{k i n}=1.25 \mathrm{GeV}$, i.e. just below the free eta meson production threshold, gave a chance to understand the $n+p$ bremsstrahlung contributions to the di-electron spectra at these low bombarding energies. Although the initial attempts based on the one-boson exchange model (OBE) to describe these results did not fully succeed, more recent OEB calculations [ए7] come close in describing the HADES $p+p$ and $n+p$ data.

\subsection{High-mass pairs from omega decays}

The high-mass di-electron pairs are of particular importance as it is expected that the study of the omega signal provides insight into in-medium vector meson modifications. The di-electron 
data in the $\mathrm{Ar}+\mathrm{KCl}$ run show a pronounced $\omega$-meson signal (see figure $\mathrm{A}$ ) and was observed for the first time at a beam energy well below the production threshold in free $\mathrm{N}+\mathrm{N}$ collisions $\left(\mathrm{E}_{t h r}^{N N}=1.89 \mathrm{~A} \mathrm{GeV}\right)$. The reconstructed signal in the $\omega$-pole mass region contains about 40 pairs, which does not allow any analysis of in-medium modifications. The signal peak position $\mathrm{M}_{\omega}=0.770 \pm 0.011 \mathrm{GeV} / \mathrm{c}^{2}$ and the width of $\sigma_{\omega}=0.022 \pm 0.010 \mathrm{GeV} / \mathrm{c}^{2}$ are determined by the intrinsic resolution of the HADES tracking system and indicate that most of the reconstructed omega candidates decayed after having left the reaction zone.

In a subsequent experiment campaign the HADES group has measured with high resolution and good event statistics inclusive di-electron invariant mass distributions in proton induced reactions on hydrogen and nuclear targets. The data for the elementary $\mathrm{p}+\mathrm{p}$ collisions at $\mathrm{E}=3.5 \mathrm{GeV}$ kinetic beam energy [एव] are compared to theoretical predictions and allow to construct a reference spectrum for the extraction and possible interpretation of effects in the $\mathrm{p}+{ }^{93} \mathrm{Nb}$ system, in particular in terms of vector meson production. An clear $\omega$-meson signal is seen in the $\mathrm{p}+{ }^{93} \mathrm{Nb}$ reaction also and preliminary results of the respective data analysis are presented in these proceedings [ए8].

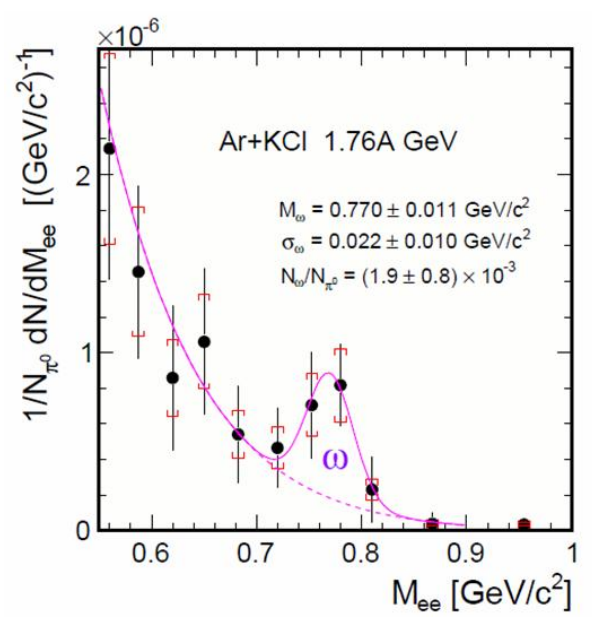

Figure 4: Dilepton invariant mass (high-mass region) distribution measured by HADES in $\mathrm{Ar}+\mathrm{KCl}$ reactions at $1.76 \mathrm{~A} \mathrm{GeV}$. For the first time in this energy region a signal of the direct omega vector meson decay, $\omega \rightarrow e^{+} e^{-}$has been observed in heavy-ion collisions. For analysis and normalization details see [W].

\section{Hadron production measured with HADES}

Although HADES was designed primarily for di-electron measurements, it has also shown an excellent capability for the identification of charged hadrons like $\mathrm{p}, \pi^{+}, \pi^{-}$and $K^{+}, K^{-}$. Hence, their production together with those of $\mathrm{K}^{0} \rightarrow \pi^{+} \pi^{-}, \Lambda \rightarrow \mathrm{p} \pi^{-}, \phi \rightarrow K^{+} K^{-}$and even $\Xi \rightarrow \Lambda \pi^{-}$has been studied in detail for the $\mathrm{Ar}+\mathrm{KCl}$ system [[20] at $\mathrm{E}=1.756 \mathrm{~A} \mathrm{GeV}$. The measured yields and slopes of the transverse mass of kaons and $\Lambda$ particles have been found to be in good agreement with the results obtained by KaoS []] and FOPI [22]. The dependencies of the measured $K^{-} / K^{+}$ ratio on the centrality and on the collision energy follow the systematics measured by KaoS [20] too. 
A combined and inclusive identification of $K^{+} K^{-}$pairs and $\phi$ mesons was performed for the first time in the same experiment at a subthreshold beam energy for $K^{-}$and $\phi$ production. The obtained $\phi / K^{-}$ratio of $0.37 \pm 0.13 \%$ indicates that $18 \pm 7 \%$ of the kaons stem from $\phi$ decays. Since the $\phi$ mesons reconstructed via the $K^{+} K^{-}$channel are those coming mainly from decays outside the nuclear medium, this value should be considered as a lower limit. In addition, nonresonant $K^{+} K^{-}$pair production can contribute to the total measured $K^{-}$yield. So far, this part is not known in heavy-ion collisions, but has been measured in elementary $\mathrm{p}+\mathrm{p}$ collisions by the ANKE collaboration [23] and contributes to about $50 \%$ of the overall $K^{+} K^{-}$yield. In this respect, a complete description of the overall $K^{-}$production in heavy-ion collisions at SIS energies requires both, the proposed strangeness exchange mechanism and the $K^{+} K^{-}$pair production processes mentioned above.

\subsection{Comparison to statistical models}

The yields of reconstructed hadron species have been extrapolated to the full solid angle and compared to the result from a fit with the statistical model THERMUS [24] as shown in Fig. []. The measured yields agree well with the results of the model, except for the $\Xi^{-}$. One should note that in this approach a good description of the $\phi$ meson yield is obtained without assuming any strangeness suppression for the $\phi$ (net strangeness content of the $\phi$ is $S=0$ ). This is very different as compared to higher energies where the $\phi$ meson does not behave as a strangeness neutral object but rather as an object with net strangeness between 1 and 2 [25]].

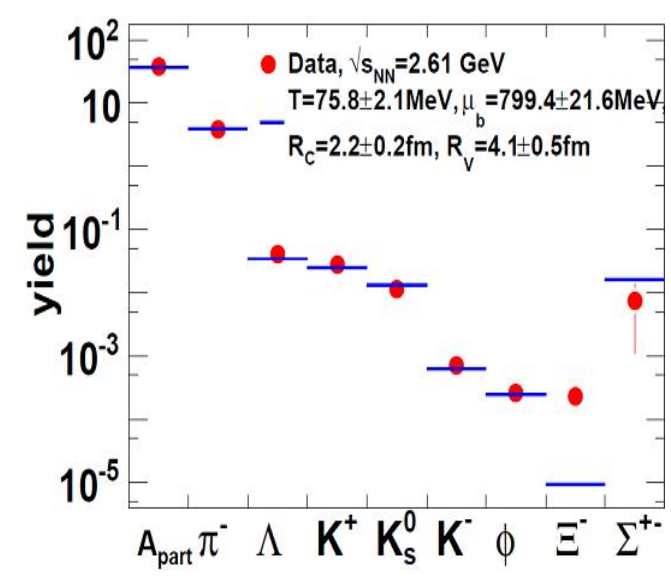

Figure 5: Hadron multiplicities (extrapolated to full solid angle) measured by HADES in Ar+KCl collisions at $1.756 \mathrm{AGeV}$ compared to the THERMUS model [24] calculations.

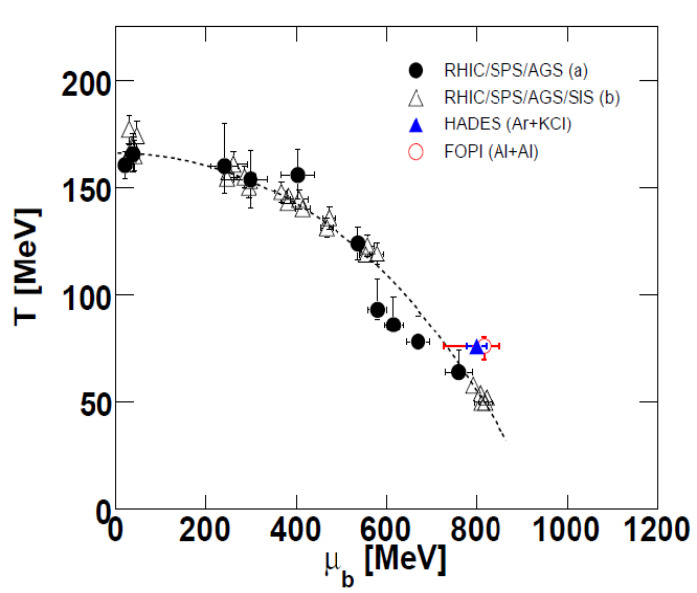

Figure 6: Chemical freeze-out parameters obtained in the statistical thermal model (for details see [B]]). The HADES point corresponds to Ar+KCl collisions at $1.756 \mathrm{AGeV}$.

The $\Xi^{-}$baryon yields measured in heavy-ion collisions above the production threshold at RHIC [26], SPS [27] and AGS [28] nicely agree with the statistical model predictions. On the contrary the result of the first $\Xi^{-}$measurement below the production threshold published by HADES [RQ], shows a deviation of about an order of magnitude from the calculations (Fig. [1). Using the 
measured hadron multiplicities, the statistical model predicts that the chemical freeze-out of the $\mathrm{Ar}+\mathrm{KCl}$ collision at $1.765 \mathrm{AGeV}$ occurs at a temperature of $\mathrm{T}=75.8 \pm 2.1 \mathrm{MeV}$ and at baryon chemical potential of $\mu=799.4 \pm 21.6 \mathrm{MeV}$. A strangeness correlation radius [32] of $\mathrm{R}_{c}=2.2 \pm 0.2 \mathrm{fm}$ was obtained, which is significantly smaller than the radius of the fireball $\mathrm{R}_{\text {fireball }}=4.1 \pm 0.5 \mathrm{fm}$.

The parameters of the obtained freeze-out point can be compared to the results from other experiments which are characterized by a striking regularity shown by particle yields at all beam energies [B]], as presented in Fig. 6. For all available energies, starting from the highest at RHIC down to the lowest at SIS18, the measured particle multiplicities are consistent with the assumption of chemical equilibrium which sets in at the end of the collision phase. Only few parameters are needed within a thermal-statistical model to describe particle yields in a very systematic way at a given collision energy. As one can also see, almost all experimental results are in good agreement with a fixed-energy-per-particle condition $\langle E\rangle /\langle N\rangle \approx 1 \mathrm{GeV}$, which is one of the available freeze-out criteria [30]].

The freeze-out point obtained from the $\mathrm{Ar}+\mathrm{KCl}$ reaction [32] slightly differs from the the striking regularity shown by particle yields, as presented in Fig. 6. A similar effect was also observed for FOPI results from $\mathrm{Al}+\mathrm{Al}$ at $1.9 \mathrm{~A} \mathrm{GeV}$. This small discrepancy can be caused by the light collision systems since these have a tendency to show higher freeze-out temperatures [ [B]].

\section{HADES at FAIR}

With beam energies available from the present SIS18 (1-2 A GeV) accelerator one can investigate nuclear matter at inverse slope parameters (temperatures) of $\mathrm{T}=40-90 \mathrm{MeV}$ and baryon densities of 1-3 $\rho_{0}$. As shown in the previous sections, the focus of the present HADES experimental programme is to perform a systematic investigation of this warm and dense region in the QCD phase diagram, utilizing meson, di-electron and hadron production in $\mathrm{N}+\mathrm{N}, \mathrm{p}+\mathrm{A}$ and $\mathrm{A}+\mathrm{A}$ collisions.

The beam energies of the planned SIS100 accelerator in future FAIR facility will allow to reach nuclear matter densities exceeding substantially those achievable at SIS18, while the fireball temperatures are expected to still remain below those estimated for the transition to the quark-gluon plasma. Exploring this region of the phase diagram is the main purpose of the proposed future HADES programme at FAIR. So far, there are no data on dilepton production available for the energy regime between 2 and $40 \mathrm{AGeV}$. Hence, this regime is "terra incognita" for di-electron measurements. From the experimental point of view, the challenges in terms of particle rates, track densities and background sources are comparable to those faced already now at SIS18 and can be accomodated by the HADES spectrometer after small detector modifications. While the present HADES set-up is operating near the light vector-meson production thresholds, the higher SIS100 energies will lead to significantly enhanced production of vector mesons. Therefore, the extension of the HADES physics programme to higher energies is a natural and very promising option.

\section{Summary}

The HADES experiment has provided systematic results on $e^{+} e^{-}$production in elementary and heavy ion collisions. The analysis of $\mathrm{C}+\mathrm{C}$ collisions at $1 \mathrm{~A} \mathrm{GeV}$ delivered high quality data and 
confirmed the old DLS findings indicating significant lack of knowledge on the dilepton production processes at these low bombarding energies. The sets of heavy ion experiments supplemented by proton induced reactions delivered a better understanding of the origin of the measured pair excess and point to the importance of low-energy processes like pion production and bremsstrahlung. The recently obtained very good agreement between measured di-electron spectra in $p+p$ and $n+p$ collisions and OBE models improved significantly the understanding of the measured data. The signal from direct $\omega$ - meson decays measured for the first time in the SIS18 energy range demonstrated the excellent performance of the HADES spectrometer in terms of mass resolution, particle identification and DAQ system.

The HADES results on strangeness production shed new light on the understanding of kaon production mechanisms in HI collisions, by providing compelling evidence that the contribution from the $\phi$ decay to the $K^{-}$yield has to be also taken into account. The measured hadron yields have in general been found in good agreement with statistical model predictions, besides the $\Xi^{-}$, which is produced far below its production threshold and shows a considerable deviation from the statistical model.

Measurements with heavier systems like $\mathrm{Au}+\mathrm{Au}$ are prepared for the near future at SIS18 and will deliver together with experiments at the planned FAIR facility new valuable data on di-electron and strangeness production. The HADES spectrometer is presently been upgraded to increase its performance and rate capabilities. An additional Forward Wall detector will allow for reaction plane reconstruction in all upcoming runs, in particular allowing to study kaon flow observables as well.

\section{Acknowledgments}

The HADES collaboration gratefully acknowledges the support by the BMBF through grants 06MT9156, 06GI146I, 06FY171, 06FY91001, and 06DR9059D (Germany), by GSI (TKrue1012, GI/ME3, OF/STR), by the Helmholtz Alliance HA216/EMMI, by the Excellence Cluster Universe (Germany), by grants GA AS CR IAA100480803 and MSMTLC 07050 MSMT (Czech Republic), by grant KBN5P03B140 20 (Poland), by INFN (Italy), by CNRS/IN2P3(France), by grants MCYT FPA2000-2041-C02-02 and XUGA PGID FPA2009-12931 T02PXIC20605PN (Spain), by grant UCY- 10.3.11.12 (Cyprus), by INTAS grant 06-1000012-8861 and EU contract RII3-CT-506078.

\section{References}

[1] C. Sturm et al. (KaoS Collaboration), Phys. Rev. Lett. 86, 39 (2001).

C. Fuchs, A. Faessler, E. Zabrodin, Phys. Rev. Lett. 86, 1974 (2001).

C. Hartnack, H. Oeschler and J. Aichelin, Phys. Rev. Lett. 96, 012302 (2006).

[2] J. Schaffner-Bielich, J. Bondorf, A. Mishustin, Nucl. Phys. A 625, 325 (1997).

W. Cassing, E. L. Bratkovskaya, U. Mosel, S. Teis, A. Sibirtsev, Nucl . Phys. A 614415 (1997).

[3] G. Agakishiev et al. Eur.Phys.J.A41:243-277,2009

[4] R.J. Porter, et al., DLS Collaboration, Phys. Rev. Lett. 79 (1997) 1229.

[5] G. Agakichiev, et al., CERES Collaboration, Eur. Phys. J. C 41 (2005) 475

D. Adamova, et al., CERES Collaboration, Phys. Rev. Lett. 91 (2003) 042301. 
[6] R. Arnaldi, et al., NA60 Collaboration, Phys. Rev. Lett. 96 (2006) 162302.

[7] H. van Hees, R. Rapp, Phys. Rev. Lett. 97 (2006) 102301.

T. Renk, J. Ruppert, Eur. Phys. J. C 49 (2007) 219

[8] C. Ernst, et al., Phys. Rev. C 58 (1998) 447.

W. Cassing, E.L. Bratkovskaya, Phys. Rep. 308 (1999) 65.

K. Shekhter, et al., Phys. Rev. C 68 (2003) 014904.

[9] G. Agakichiev, et al., HADES Collaboration, Physics Letters B 663 (2008) 43-48.

[10] G. Agakichiev et al. HADES Collaboration, Phys. Rev. Lett. 98 (2007) 052302

[11] G. Agakichiev et al. HADES Collaboration,. e-Print: arXiv:1103.0876 [nucl-ex]

[12] R. Averbeck et al. (TAPS Collab.), Z. Phys. A 359, 65 (1997).

[13] R. Averbeck, R. Holzmann, V. Metag, and R. S. Simon, Phys. Rev. C 67, 024903 (2003).

[14] G. Agakishiev et al. (HADES Collab.), Phys. Lett. B 690, 118 (2010).

[15] L. P. Kaptari and B. Kíampfer, Nucl. Phys. A 764, 338 (2006), Phys. Rev. C 80, 064003 (2009).

[16] R. Shyam and U. Mosel, Phys. Rev. C 67, 065202 (2003).

[17] R. Shyam and U. Mosel, arXiv:1006.3873v1

[18] XLIX International Winter Meeting On Nuclear Physics, 24-28 Jan 2011, Bormio, Italy

[19] 6th International Workshop on Critical Point and Onset of Deconfinement, 23 - 29 August 2010, Dubna, Russia

[20] G.Agakichev et al., (HADES), in press Phys. Rev. C and arXiv:0902.3487

[21] A. Förster et al. (KAOS) Phys. Rev. C 75 (2007) 024906.

[22] M. Merschmeyer et al. (FOPI) Phys. Rev. C 76 (2007) 024906

[23] Y.Maeda et al. (ANKE), Phys. Rev. C 77 (2008) 015204

[24] S. Wheaton and J. Cleymans, hep-ph/0407174

S. Wheaton and J. Cleymans, J. Phys. G31 (2005) S1069

[25] I. Kraus et al., Phys. Rev. C76, 064903 (2007)

[26] J. Adams et al. (STAR), Phys. Rev. Lett. bold98, 062301 (2007)

[27] F. Antinori et al. (NA57) Phys. Lett. B 595, 68 (2004)

C. Alt et al. (NA49), Phys. Rev. C 78, 034918 (2008)

[28] P. Chung et al. (E895), Phys. Rev. Lett. 91, 202301 (2003)

[29] G. Agakishiev et al. (HADES) Phys. Rev. Lett. 1003 (2009) 132301

[30] J. Cleymans at al. Phys. Rev. C 73, 034905 (2006) and J. Cleymans private communication

[31] J. Cleymans et al., Phys. Rev. C 59, 1663 (1999).

[32] G. Agakishiev et al. Eur. Phys. J. A 47:21, 2011 